IPHE NOTE 2000-020

November 24, 2000

Proceedings of the $V I$ international workshop on tau physics

18-21 September 2000, Victoria, Canada

to be published in Nuclear Physics (Proc. Suppl.)

\title{
First study of LHCb sensitivity to neutrinoless $\tau$ decays
}

Paolo Bartalini ${ }^{a}$ on behalf of the LHCb collaboration

a) Institut de Physique des Hautes Energies (IPHE), Université de Lausanne

\begin{abstract}
A first estimate of the $\tau$ physics potential at LHCb is presented, concentrating on the search for $\tau \rightarrow \mu \mu \mu$ decays. If no signal is observed after 1 year of data taking, LHCb is expected to be able to set a $90 \%$ C.L upper limit of $1.8 \times 10^{-7}$ on the corresponding branching ratio.
\end{abstract}




\section{Introduction}

This document is divided in 8. sections. The LHCb detector is introduced in section 2., with particular emphasis on trigger performances. $\tau$ production at the LHC is briefly reviewed in section 3., focusing on the importance of non-prompt production from charm and beauty hadrons decays, which constitute the main source of $\tau$ leptons at LHCb. In section 4. the status of the art of neutrinoless $\tau$ decays is summarised. The possibility to study neutrinoless $\tau$ decays at LHCb is discussed in section 5., concentrating on the reference channel $\tau \rightarrow \mu \mu \mu$. The experimental detection methods are described in section 6. where the estimation of the selection efficiencies for both signal and background events is also reported. The expected sensitivity and the possible exclusion limits (under the hypothesis of no observation at $\mathrm{LHCb}$ ) are quoted in section 7.. The overall imulation results are summarised in the conclusions (section 8.), where the possibility to refine the present conservative simulation results is also discussed.

\section{The LHCb experiment}

The LHCb experiment is described in the Technical Proposal [1]. The LHCb detector is a single arm spectrometer (figure 1) to be installed at the Large Hadron Collider (LHC), covering the pseudorapidity range $1.8<\eta<4.9$, in the forward region of the proton-proton interactions. In order to reduce the number of multiple pp interactions per bunch crossing (pile-up) and to limit the occupancy and the radiation dose absorbed by the detectors, $\mathrm{LHCb}$ is planning to run at an average tuneable luminosity of $2 \times 10^{32} \mathrm{~cm}^{-2} \mathrm{~s}^{-1}$. Since the $c \bar{c}$ and $b \bar{b}$ production at the LHC will take place predominantly at large pseudorapidity, the forward geometry of the LHCb detector will allow to collect a considerable statistics of charm and beauty hadrons. As shown in section 3., a significant production of $\tau$ leptons in the LHCb acceptance has to be expected as well.

The vertex locator (VELO), that surrounds the interaction region, is made of 25 stations of $200 \mu \mathrm{m}$ silicon sensors with $\mathrm{r}$ and $\phi$ strips. The VELO is delegate to measure precisely the position of the primary and secondary vertices and to provide tracking information to the level 1 trigger.

Two RICH detectors [2] achieve a kaon/pion separation better than three sigma in the momentum range between 2 and $100 \mathrm{GeV} / \mathrm{c}$.

The tracking system (TRACK), consisting of 11 stations and a warm dipole magnet [3] producing a vertical field of $4 \mathrm{Tm}$, will achieve a $0.3 \%$ momentum resolution on average for tracks up to $200 \mathrm{GeV} / \mathrm{c}$. The typical mass resolution performance of the tracking system is estimated on the reference channel $B^{0} \rightarrow \pi^{-} \pi^{+}$, for which $17 \mathrm{MeV} / \mathrm{c}^{2}$ is quoted.

The LHCb calorimeter system [4] is made of a lead scintillator preshower, a $25 X_{0}$ shashlik type ECAL and a 5.6 $\lambda \mathrm{Fe}+$ scintillating tiles HCAL. The expected energy resolutions are $\sigma_{E} / E=$ $9.5 \% / \sqrt{E} \oplus 1 \%$ for the ECAL and $\sigma_{E} / E=80 \% / \sqrt{E} \oplus 10 \%$ for the HCAL. The main purpose of the calorimeter system is to provide identification and kinematics of electrons, photons and hadrons for the level 0 trigger.

The muon detector (MUON) is composed of 5 stations, based on resistive plate chambers and multi-wire proportional chambers for the central high rate region; it provides identification and kinematics of muons for the level 0 trigger.

The LHCb trigger system is organised in a flexible scheme that comprises 4 different levels.

The level 0 (L0) trigger selects events with hadrons and leptons that have transverse momentum greater than 2 or $3 \mathrm{GeV}$. A pile-up veto discards events with multiple pp interactions in one bunch crossing. The L0 trigger has an input frequency of $40 \mathrm{MHz}$, an output frequency of $1 \mathrm{MHz}$ and a latency of about $4 \mu \mathrm{s}$.

The level 1 (L1) algorithm uses the VELO information and reconstructs three dimensional vertices from tracks with high impact parameter in the $\mathrm{r}-\phi$ projection. The L1 trigger is optimised to select the $b$ events among the minimum bias pp interactions accepted by the L0 trigger and is characterised by a 


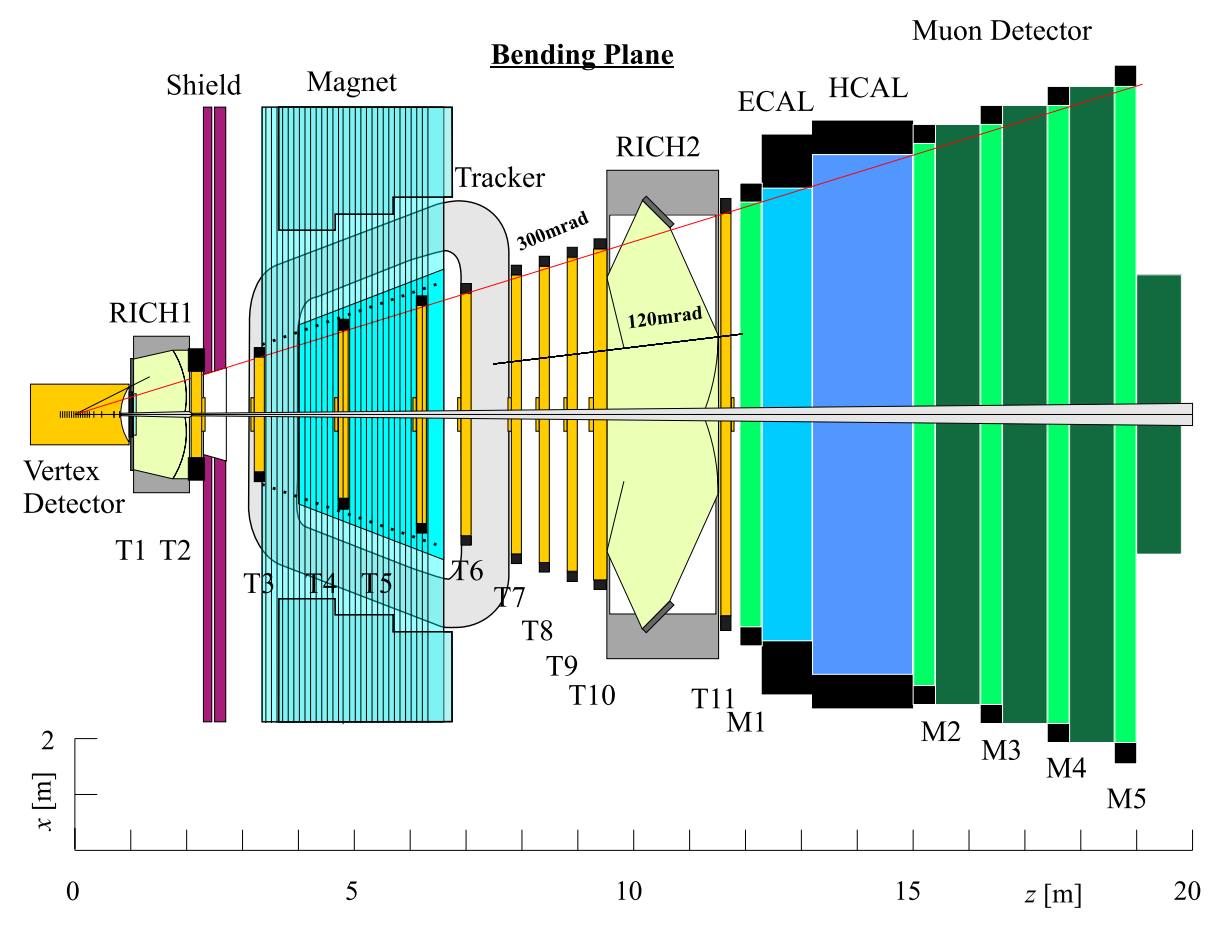

Fig. 1: Horizontal view of the LHCb detector .

suppression factor of 25 and a latency of about $1 \mathrm{~ms}$.

In some particular studies of exotic events characterised by a clear topology, as in the case of multi-muon patterns, a possibility to relax or bypass the L1 trigger requirement is taken into account in the design, allowing to keep high physics performance in many searches of Standard Model forbidden decays of charm hadrons and $\tau$ leptons.

The level 2 (L2) and the level 3 (L3) triggers, provide the complete reconstruction of final states and have access to the full event information from all the subdetectors; they are characterised by suppression factor of 8 and 25 respectively, which reduce the rate down to about $200 \mathrm{~Hz}$.

\section{Production of $\tau$ leptons at the LHC}

For $\tau$ production studies at the LHC, Pythia 6.1 [5] with the parton shower option, the CTEQ4L parton distribution functions and the tuning published in $[6,7]$ is adopted as reliable QCD model, while QQ 9.2 [8], that currently contains the best known routines and the most up-to-date decay table for charm and beauty hadrons, is used as decay model. According to the Pythia predictions the total inelastic cross section for proton proton interactions at $\sqrt{s}=14 \mathrm{TeV}$ is assumed to be $\sigma_{\text {inel }}=80 \mathrm{mb}$, while $\sigma_{n d}=55 \mathrm{mb}$ is considered for the total non-diffractive inelastic cross section.

Table 1: $\tau$ production branching fractions and relative $\tau$ cross section contributions for charm and beauty mesons as predicted by Pythia 6.1 and QQ 9.2.

\begin{tabular}{llllll}
\hline Meson $(\mathrm{M})$ & $D_{s}\left(\bar{D}_{s}\right)$ & $D^{+}\left(D^{-}\right)$ & $B^{0}\left(\bar{B}^{0}\right)$ & $B_{s}\left(\bar{B}_{s}\right)$ & $B^{+}\left(B^{-}\right)$ \\
\hline $\operatorname{Br}(M \rightarrow \tau+X)$ & $7.0 \%$ & $0.2 \%$ & $2.7 \%$ & $1.5 \%$ & $2.7 \%$ \\
\hline$\sigma(p p \rightarrow M \rightarrow \tau+X) / \sigma(p p \rightarrow \tau+X)$ & $77 \%$ & $3 \%$ & $9 \%$ & $2 \%$ & $9 \%$ \\
\hline
\end{tabular}



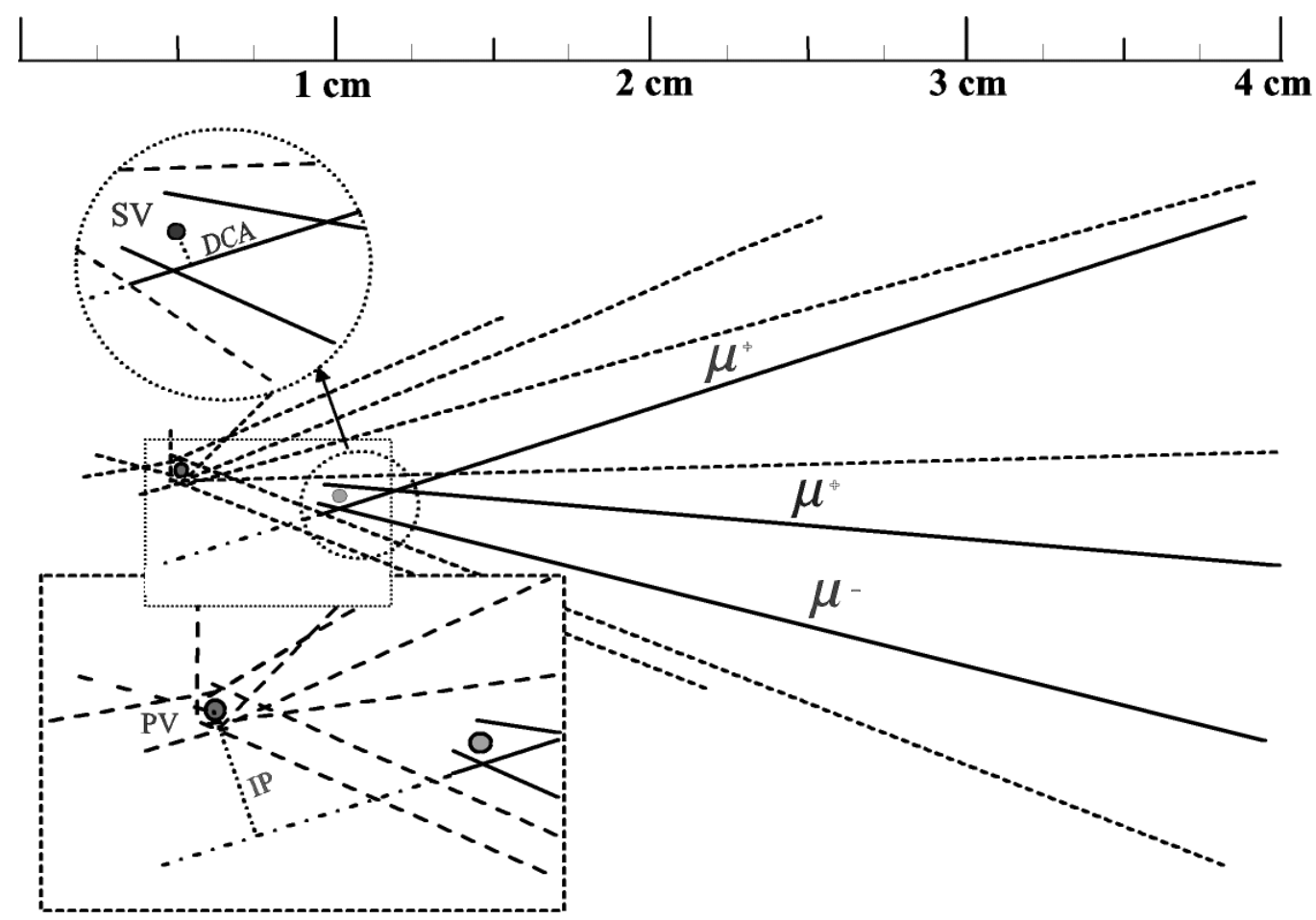

Fig. 2: Sketch of a typical $\tau \rightarrow \mu \mu \mu$ decay reconstructed in the forward LHCb geometry; the length scale is reported on top; the rectangular and the round frames show the zoomed details around the reconstructed primary vertex (PV) and secondary vertex (SV) respectively; DCA indicates the distance of closest approach of th muon candidate with respect to the SV while IP indicates the impact parameter of the muon with respect to the PV.

In such configuration for the physics Monte Carlo simulations the $\tau$ inclusive production cross section turns out to be $\sigma(p p \rightarrow \tau+X) \simeq 120 \mu b$, while the $\tau$ - $\tau$ inclusive production cross section turns out to be $\sigma(p p \rightarrow \tau+\tau+X) \simeq 2 \mu b$. About $77 \%$ of the $\tau$ leptons are produced in $D_{s}$ meson leptonic decays while the rest arise basically from $c$ and $b$ semileptonic decays. The details of the simulation results are summarised in table 1 . Note that the prompt $\tau$ production is most likely to be negligible compared to the one from long-lived particle decays, for instance $\sigma(p p \rightarrow W \rightarrow \tau \nu) \simeq 15 n b$.

Considering the nominal average luminosity of $2 \times 10^{32} \mathrm{~cm}^{-2} \mathrm{~s}^{-1}$, the total annual $\tau$ yield is estimated to be about $2.5 \times 10^{11}$, of which $0.7 \times 10^{11}$ in the LHCb acceptance $(1.8<\eta<4.9)$.

One of the most critical values used for the $\tau$ event yield estimate reported in this paper is the branching fraction of the leptonic $D_{s}$ meson decay into the $\tau \nu$ doublet, for which the best experimental result is currently $\operatorname{Br}\left(D_{s} \rightarrow \tau \nu_{\tau}\right)=7 \pm 4 \%$, measured by the L3 experiment [9]. From a theoretical point of view the purely leptonic $D_{s}$ meson decay is a classical problem that can be treated following the lines of the leptonic pion decay, the main source of uncertainty being the decay constant $f_{D_{s}}$. A recent derivation can be found in [10] where $\operatorname{Br}\left(D_{s} \rightarrow \tau \nu_{\tau}\right)=6.3 \pm 0.5 \%$ is quoted. It should be noticed that the uncertainties on the heavy flavour production cross sections affects the $\tau$ event yield estimation as well; from a theoretical point of view we can consider a relative error of $30 \%$ on the absolute Pythia 6.1 predictions, that receives contributions from uncertainties in both the total cross sections and the amount of gluon splitting contributing to the heavy quark production; however these quantities will be much more precisely known as soon as the first pp collisions will be measured by the LHC experiments. 
Table 2: Estimated selection efficiencies of signal and background events reported for Preliminary, Identification, Topology, Mass, Trigger and Total cuts. The signal cross section and the event yield are quoted at the current 90\% C.L. limit [12] for the corresponding branching ratio: $\operatorname{Br}(\tau \rightarrow \mu \mu \mu)=1.9 \times 10^{-6}$.

\begin{tabular}{llll}
\hline & signal & $b \rightarrow \mu X$ & $b \bar{b}$ \\
\hline$\sigma$ & $(0.25 \mathrm{nb})$ & $0.100 \mathrm{mb}$ & $0.500 \mathrm{mb}$ \\
Preliminary & $6.6 \%$ & $7.4 \%$ & $3.0 \%$ \\
Identification & $84 \%$ & $5.8 \%$ & $3.3 \%$ \\
Topology & $57 \%$ & $1.7 \%$ & $1.9 \%$ \\
Mass & $98 \%$ & $0.6 \%$ & $0.7 \%$ \\
Trigger & $22 \%$ & $54 \%$ & $43 \%$ \\
Total & $0.70 \times 10^{-3}$ & $2.7 \times 10^{-7}$ & $0.60 \times 10^{-7}$ \\
$N_{\text {1year }}$ & $(3200)$ & 52000 & 60000 \\
\hline
\end{tabular}

\section{Neutrinoless $\tau$ decays}

Not all the $\tau$ physics is open to LHCb. However, in order to reduce the effect of combinatorial background, LHCb can concentrate on the study of neutrinoless $\tau$ decays, in which the $\tau$ lepton mass can be reconstructed with sufficient resolution.

Neutrinoless decays with lepton flavour violation are expected in many extensions of the Standard Model [11], including GUT's, Left-Right symmetric models, SUSY with broken R-parity, Supergravity theories, Technicolor etc. and several calculated rates are very close to the present experimental limits [12]. For example there are models that predict branching fractions for the decay of a $\tau$ lepton in three muons as large as $10^{-6}$, while the current limit, set by the CLEO experiment, is $\operatorname{Br}(\tau \rightarrow \mu \mu \mu)<1.9 \times 10^{-6}$ at $90 \%$ confidence level.

\section{Motivations to study $\tau \rightarrow \mu \mu \mu$ decays at $\mathrm{LHCb}$}

The first feasibility study of a search for neutrinoless $\tau$ decays at LHCb is performed on the $\tau \rightarrow \mu \mu \mu$ channel; the simple event topology that characterises such kind of events at detector level, in which three reconstructed muon candidates converge in a common displaced secondary vertex, is sketched in figure 2 .

This channel has a lot of advantages: muons are easy to be triggered and reconstructed with a good momentum resolution. Since there is no missing particle, the $\tau$ mass should be reconstructed with an excellent resolution.

On top of that, as most of the $\tau$ leptons are produced in charm and beauty hadrons decays (see section 3.), LHCb should preserve a sufficiently high (though not optimised) L1 trigger efficiency even running the trigger system in standard conditions (see section 2.).

As shown in figure 3, due to the high relativistic boost of the $\tau$ leptons produced at the LHC, a strong correlation between the muons and $\tau$ pseudorapidities has to be expected when $\tau$ leptons decay into three muons. It is estimated that about $16.3 \%$ of the $\tau \rightarrow \mu \mu \mu$ events produced at LHC have all the three muons in the LHCb acceptance region $(1.8<\eta<4.9)$.

\section{Detection of $\tau \rightarrow \mu \mu \mu$ decays at $\mathrm{LHCb}$}

For physics performance studies, events are produced by Pythia 6.1 in conjunction to QQ 9.2, with the configuration described in section 3.. The simulation of the $\mathrm{LHCb}$ detector response is based on the GEANT 3.21 package. In order to reconstruct the three muons candidates converging in a common 


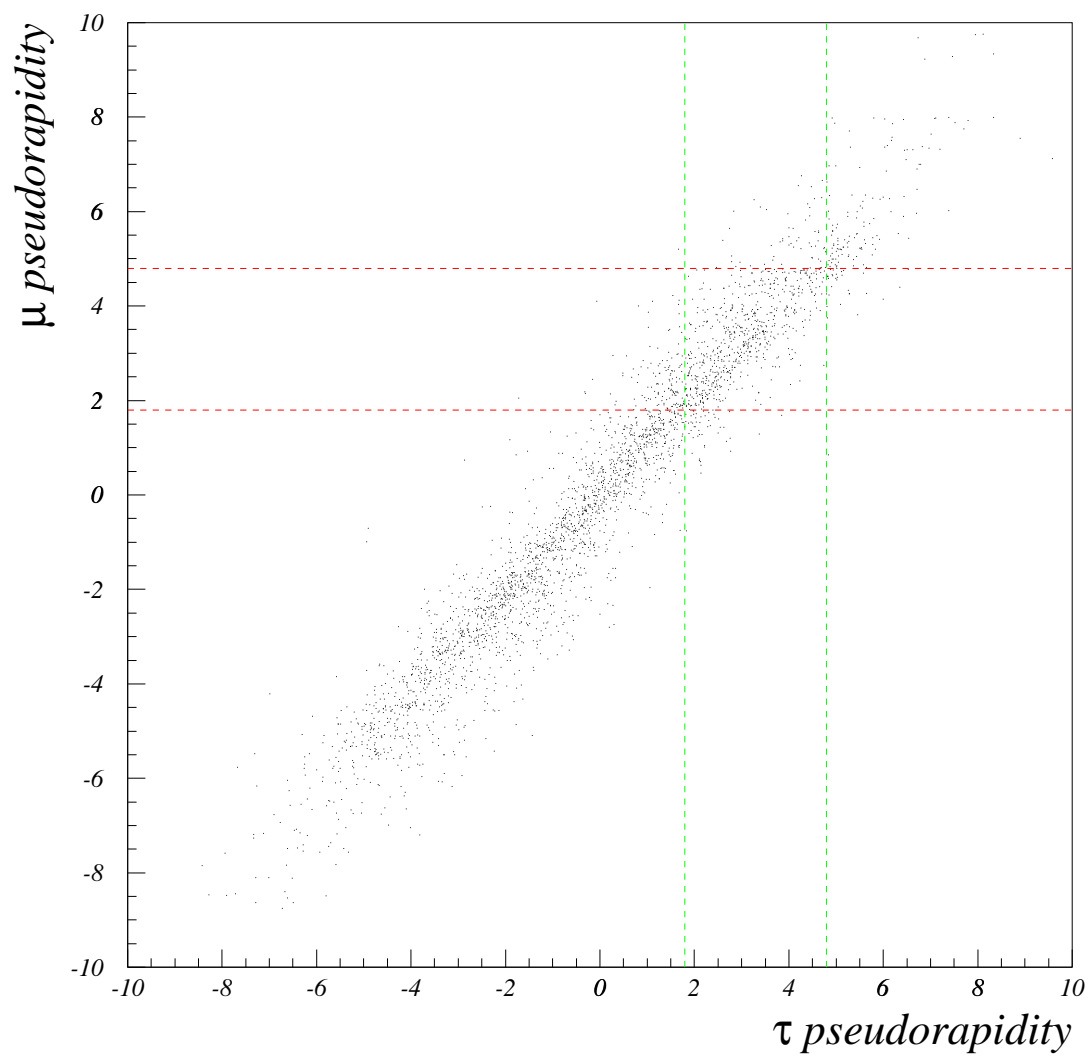

Fig. 3: $\mu$ pseudorapidity versus $\tau$ pseudorapidity in $\tau \rightarrow \mu \mu \mu$ decays at LHC; the LHCb pseudorapidity range $(1.8<\eta<4.9)$ is indicated by the horizontal and vertical lines.

secondary vertex, four different set of selections are applied: the Identification cuts have the purpose to select the muons candidates among the reconstructed charged tracks; the Topology cuts require the muon candidates to form a secondary vertex well separated from the primary vertex; the Mass cuts select a reconstructed mass window centered on the nominal $\tau$ mass; the Trigger cuts comprise the basic requests to pass the L0 and L1 triggers and the presence of at least one triggering muon. More detailed explanations of the four different selection follow.

\section{Identification cuts:}

- charged tracks must have matching hits in VELO, RICH, TRACK and MUON detectors;

- charged tracks have to be best identified as $\mu^{ \pm}$with C.L.>90\%;

- charged tracks must have a reconstructed momentum $P>3 \mathrm{GeV}$;

- only those combinations of three charged tracks with total charge \pm 1 are taken into account. Topology cuts:

- the three muon candidates originate from a common secondary vertex with a $\chi^{2}$ probability $P\left(\chi^{2}\right)>$ $2 \%$

- each muon candidate is required to have a distance of closest approach $D C A<100 \mu m$ with respect to the reconstructed secondary vertex;

- each muon candidate is required to have an impact parameter significance $I P / \Delta(I P)>1.5$ with respect to the reconstructed primary vertex. 


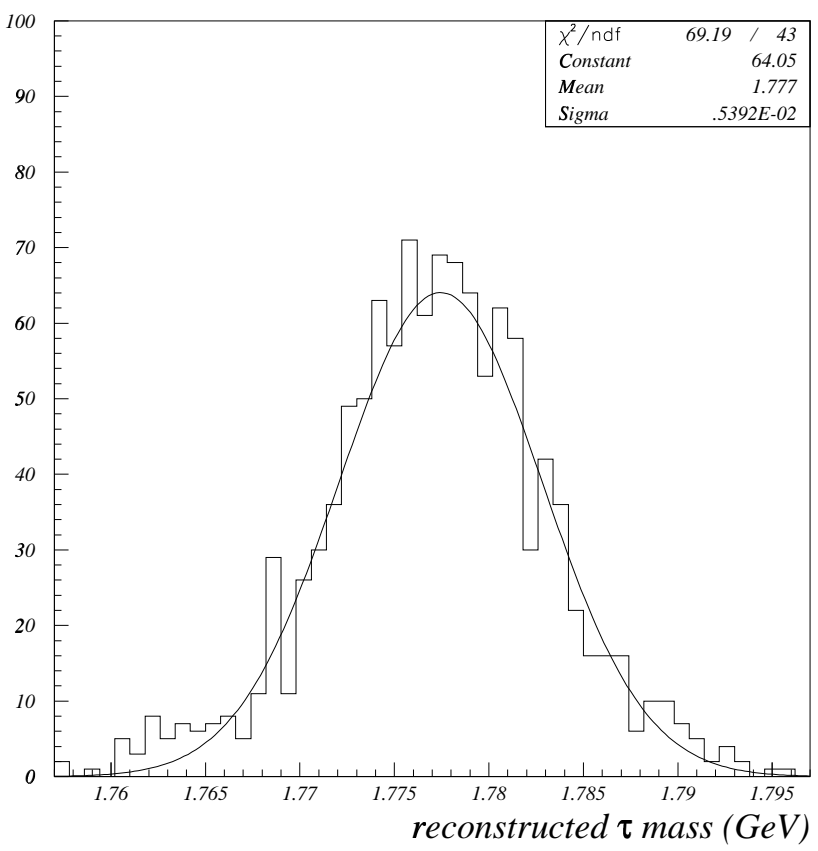

Fig. 4: $\tau$ mass distribution for $\tau \rightarrow \mu \mu \mu$ events; the superimposed gaussian fit is also shown.

\section{Mass cuts:}

- the reconstructed $\tau$ mass has to be in a window of $\pm 20 \mathrm{MeV}$ around the nominal $\tau$ mass $M(\tau)=$ $1777 \mathrm{MeV}$.

Trigger cuts:

- the event must pass the L0 trigger;

- the event must pass the L1 trigger;

- at least one muon candidate has to have a transverse momentum $P_{T}>1.0 \mathrm{GeV}$.

The mass distribution for signal events is shown in figure 4, the corresponding mass resolution turns out to be $5.4 \mathrm{MeV}$.

In principle one should estimate the background efficiency of the overall selection described above applying in sequence the sets of Identification, Topology, Mass and Trigger cuts. In practice this procedure cannot be adopted for most of the generic backgrounds ( $\tau$ inclusive, $b$ inclusive, $c$ inclusive etc.) because of the limited available Monte Carlo statistics: in order to estimate reliable selection efficiencies as low as $10^{-8} \div 10^{-7}$, a sample of 10 to 100 millions of events would be needed. The realistic assumption of background rates locally proportional to the mass window width allows to reduce such needs by a factor 25, evaluating the rates on the enlarged mass window of $\pm 500 \mathrm{MeV}$ and rescaling them to the $\pm 20 \mathrm{MeV}$ one, but, even so, and since no specific exclusive background has been identified, the needed Monte Carlo statistics turns out to be still very high with respect to what LHCb has generated so far. That's why a further trick is needed that consist in introducing some Preselection cuts as looser Identification cuts, defined as follows.

\section{Preselection cuts:}

- charged tracks must have matching hits in VELO, RICH, TRACK and MUON detectors; 


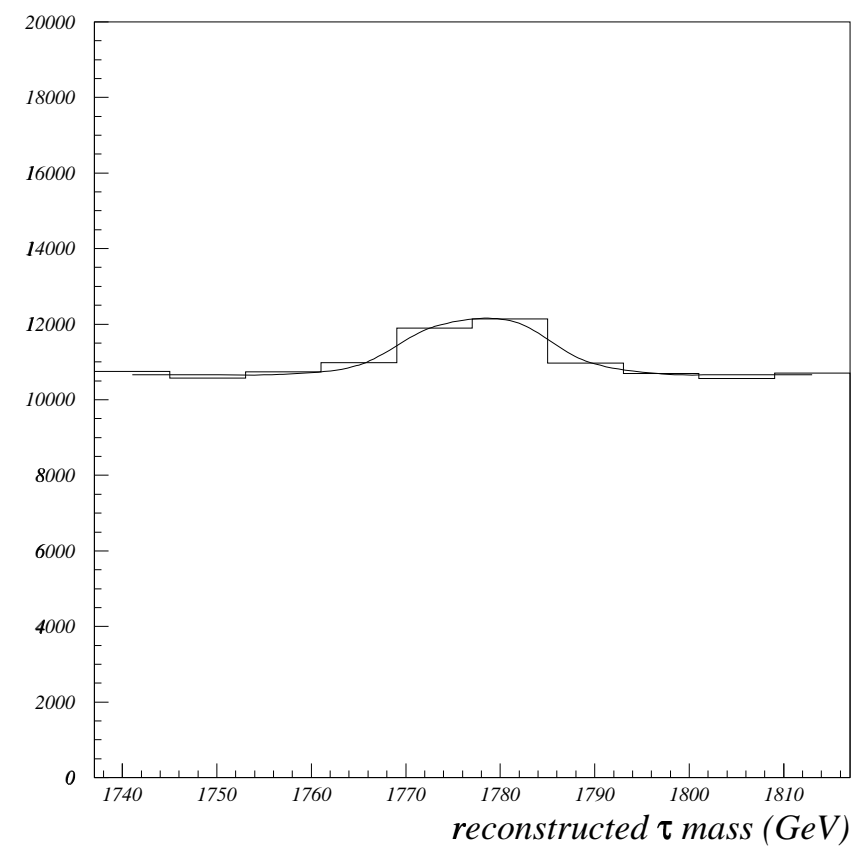

Fig. 5: Mass distribution of reconstructed $\tau \rightarrow \mu \mu \mu$ candidates; the signal is produced assuming $\operatorname{Br}(\tau \rightarrow \mu \mu \mu)=1.9 \times 10^{-6}$; the high statistics background is generated by a toy Monte Carlo and is considered flat in the histogram mass window; the overall statistics corresponds to 1 year data taking; the superimposed gaussian + constant fit is also shown.

- charged tracks have to be best identified as $\mu^{ \pm}$with C.L.> 30\%;

- charged tracks must have a reconstructed momentum $P>1 \mathrm{GeV}$;

- only those combinations of three charged tracks with total charge \pm 1 are taken into account.

The efficiencies relative to the Identification, Topology, Mass and Trigger cuts are then redefined with respect to the events that pass the Preselection cuts and the Total selection efficiencies are estimated under the factorisation assumption:

$$
\epsilon_{\text {Total }}=\epsilon_{\text {Preselection }} \times \epsilon_{\text {Identification }} \times \epsilon_{\text {Topology }} \times \epsilon_{\text {Mass }} \times \epsilon_{\text {Trigger }}
$$

However the true total selection efficiencies would be the one obtained in the limit Preselection cuts $\rightarrow$ Identification cuts, but the cross checks made on the signal events show that the method described above produce meaningful results, bringing in average to an overestimation of about $30 \%$ for the overall selection efficiency, therefore it turns out to be a very conservative approach in background estimations. Some improvements are expected as soon as more background events will be available. From an heuristic point of view, we can account for the increased overall selection efficiencies considering that the looser muon Identification cuts tends to select more true pions that compared to the true muons are more frequently associated to common secondary particles.

The selection efficiencies and the expected event yields for signal events and those obtained assuming that all the cuts are uncorrelated for $b \rightarrow \mu X$ semileptonic and $b \bar{b}$ inclusive events, are reported in table 2. Different kinds of background such as $\tau$ inclusive, and $c \bar{c}$ inclusive decays have also been tried but have been demonstrated to be less important than those coming from $b \rightarrow \mu X$. The very low trigger efficiency being the main reduction factor for $\tau$ and $c \bar{c}$ decays. From the Total efficiencies row, it results that the $b \bar{b}$ background is compatible with the $b \rightarrow \mu X$ one, hence there should be no further 
Table 3: Detail of the Trigger cuts efficiencies for signal, $b \rightarrow \mu X$ and $b \bar{b}$ events

\begin{tabular}{llll}
\hline & signal & $b \rightarrow \mu X$ & $b \bar{b}$ \\
\hline LO Trigger & $82 \%$ & $93 \%$ & $92 \%$ \\
L1 Trigger & $34 \%$ & $64 \%$ & $53 \%$ \\
$P_{T}$ min. & $81 \%$ & $92 \%$ & $88 \%$ \\
Total Trigger & $22 \%$ & $54 \%$ & $43 \%$ \\
\hline
\end{tabular}

major source of background among the $b \bar{b}$ events; a detailed analysis performed on those $b \bar{b}$ events that pass at least three out of the four set of cuts confirms such hypothesis.

From a preliminary study of those events that pass at least Identification and some looser Topology cuts, it is found that most of the background consists of a true muon combined with random tracks (combinatorial background). There's some contamination due to di-muons from $\eta\left(\eta^{\prime}\right) \rightarrow \mu^{+} \mu^{-} \gamma$ and $\omega \rightarrow \mu^{+} \mu^{-} \pi^{0}$, but these have small branching fractions in the range $10^{-4}-10^{-3}$ and are taken into account in the QQ 9.2 decay table.

As seen in table 2, the Trigger efficiency for the signal events is found to be much lower than that the background events; details of the Trigger cut efficiencies for signal and background events are reported in table 3 and show that the main reduction factor on signal events is caused by the poor $L 1$ Trigger efficiency. However the L1 trigger is still not optimised for $\tau$ physics but the characteristics peculiar to the $\tau \rightarrow \mu \mu \mu$ decay allows to consider the alternative to bypass the L1, introducing a low rate multi-muon trigger at L0; this possibility will be taken into account in future studies.

\section{Sensitivity and possible exclusion limits}

With the current very conservative estimation of the LHCb performances, and under the assumption of no observation of $\tau \rightarrow \mu \mu \mu$, it will be possible to set a limit of $1.8 \times 10^{-7}$ on $\operatorname{Br}(\tau \rightarrow \mu \mu \mu)$ after 1 year of data taking. The limit will be improved to $0.8 \times 10^{-7}$ in 5 years. On the other hand, as shown in figure 5, assuming an event yield corresponding to the current $90 \%$ C.L. limit [12], the signal peak can be clearly seen in the mass distribution of reconstructed $\tau \rightarrow \mu \mu \mu$ candidates. A 5 sigma discovery signal will be detected after 1 year of data taking if $\operatorname{Br}(\tau \rightarrow \mu \mu \mu)>1.1 \times 10^{-6}$.

\section{Conclusions}

We have shown that the $\tau$ yield in the LHCb acceptance is about $0.7 \times 10^{11}$ per year, the main production mechanism arising from the $D_{s}$ meson leptonic decays.

A $\tau \rightarrow \mu \mu \mu$ search at LHCb, based on the selection of secondary vertices formed by three muon candidates, is feasable. The $b \rightarrow \mu X$ events have been identified as the main background source. From the first conservative estimation of signal and background efficiencies, under the assumption of no observation at $\mathrm{LHCb}$, we conclude that it will be possible to set a limit of $1.8 \times 10^{-7}$ at $90 \%$ C.L. on $\operatorname{Br}(\tau \rightarrow \mu \mu \mu)$ after 1 year of data taking $\left(0.8 \times 10^{-7}\right.$ at $90 \%$ C.L. after 5 years $)$. However these simulation results need to be refined. Some improvements are expected from the L1 trigger optimisation and from releasing the factorisation hypothesis, as soon as larger Monte Carlo statistics will be available to LHCb.

Further feasibility studies will be soon extended to neutrinoless $\tau$ decays with $\pi$ and $K$ mesons in the final state. 


\section{References}

[1] S. Amato et al. (LHCb collaboration), LHCb Technical Proposal, CERN/LHCC 98-4, LHCC/P4.

[2] S. Amato et al. (LHCb collaboration), LHCb RICH technical design report, CERN/LHCC 2000-037.

[3] S. Amato et al. (LHCb collaboration), LHCb magnet technical design report, CERN/LHCC 2000007.

[4] S. Amato et al. (LHCb collaboration), LHCb calorimeters technical design report, CERN/LHCC 2000-036.

[5] T. Sjöstrand, P. Eden, C. Friberg, L. Lönnblad, G. Miu, S. Mrenna and E. Norrbin, hep-ph/0010017.

[6] P. Nason G. Ridolfi, O. Schneider, G.F. Tartarelli, P. Vikas (conveners) et al., Standard Model physics (and more) at the LHC, Bottom production, Yellow report CERN 2000-004, hep-ph/0003142

[7] P. Bartalini, J. De Mello, P. Koppenburg, T. Nakada, O. Schneider, LHCb PHYS 99-028.

[8] CLEO Collaboration, QQ Documentation, http://www.lns.cornell.edu/public/ CLEO/soft/QQ/qq_expert.html.

[9] M. Acciarri et al. (L3 Collaboration), Phys.Lett.B 396 (1997) 327.

[10] R. Schwienhorst, Ph.D. thesis, University of Minnesota (2000).

[11] R. Stroynowski, Nucl.Phys.B (Proc. Suppl.) 76 (1999) 185.

[12] D. W. Bliss et al. (CLEO Collaboration), Phys.Rev.D 57 (1998) 5903. 